\title{
How we talk about the movies: A comparison of Australian, British and American film genre terms
}

\begin{abstract}
Vocabulary or terminological control has been an issue of critical information practice for Australian information professionals for many years. In the 1970s Australian libraries began to supplement Library of Congress Subject Headings with their own List of Australian Subject Headings (McKinlay, 1979), and today there remains the bibliographic need to cover uniquely Australian terms and concepts, including those about Indigenous Australian culture (Hider, 2019, p.13). The library world is not the only domain, however, to have developed vocabularies to describe and make sense of information resources. Comparison of film genre vocabularies is of particular interest because film studies have often assumed a fixed set of categories, regardless of geography, culture or time. Although much of today's film industry is "global", with a strong Hollywood influence on genre to sell movies, this does not mean that filmmakers, nor film audiences, use a set vocabulary. This paper looks at whether similar geographical biases may be discerned in vocabularies used in the domain of film curation by examining the variation in terminology and the classification of film genres used by film institutes based in Australia, the United Kingdom and the United States.
\end{abstract}

Keywords: terminology control; film genre; cross-cultural vocabulary usage

\section{Introduction}

The creation and use of controlled vocabularies (such as subject heading lists, subject thesauri, term lists and taxonomies) have preoccupied considerable numbers of information professionals for well over a hundred years, while the need for these vocabularies to accommodate particular cultural perspectives has been identified on multiple occasions throughout this period, including in Australia. These perspectives can influence the concepts represented in the vocabularies, the way these concepts are structured, and also the terms used for the concepts. For example, in the last century, local extensions of the Dewey Decimal Classification scheme were devised and adopted for Australian topography and literature, while the United States-centric Library of Congress Subject Headings (LCSH) were supplemented by the List of Australian Subject Headings(LASH) (McKinlay, 1979) to cover various concepts and terms of importance to Australia library users (Hider, 2019). Even today, in our globalised world, there remains a bibliographic need for some localised vocabulary control. For example, many concepts of Indigenous Australian culture are still not included in the international vocabularies (Hider, 2019, p.13).

The library world is not the only domain that has developed vocabularies to describe and make sense of information resources, nor the only one in which culture is a factor in this development. Another such world is that of curation, which of course has some overlap with librarianship, including resources collected and described. As well as overlap, however, there are also differences of perspectives, approaches, traditions, and so forth. The article considers how such differences, in the specific subdomain of film curation, affect, or do not affect, the influence of culture on resource description and the resulting vocabularies. Such a study is of particular interest as film studies have often assumed a fixed set of categories in relation to 'genre', regardless of geography, culture 
or time. Has this assumption limited culture as a factor on the vocabularies of film institutes in different parts of the world?

The research reported in this article is part of a broader project examining the universality, or particularism, of film genres across domains and cultures, another part of which is reported by Hider, White and Barlow (forthcoming). This article focuses on the specific domain of film curation and compares the formal genre classifications employed by film institutes in three different English-speaking countries, based in Australia, the United Kingdom and the United States. Both the concepts and terms included in these vocabularies are analysed.

\section{Literature Review}

As Hider, White and Barlow (forthcoming) point out, films are classified and described according to 'genre' in a variety of different communities, including but not limited to social media, film education, film preservation, and library and information science. For English speaking countries Library of Congress-created vocabularies have a long history of use and implementation. Film genres were originally a part of the Library of Congress Subject Headings (LCSH) but starting in 2007 film genres started to be separated into a new vocabulary that focused on genres and forms of material, as opposed to their subjects (Young and Mandelstam, 2013). The Library of Congress Genre Formats and Terms (LCGFT) (Library of Congress, 2019) covers a wide range of materials and domains in which these materials are found (and collected by the Library of Congress and other libraries). According to the LCGFT (2019, p.3), 'Genres and forms may be broadly defined as categories of resources that share known conventions'. Given this broad library community-based definition, the LCGFT contains 206 terms under the heading 'motion pictures', otherwise known as 'films' (Hider, White \& Barlow, forthcoming). Many of these terms align with earlier genre lists used by film librarians, including the Moving Image Genre-Form Guide (MIGFG) and the Moving Image Materials: Genre Terms (MIM) (Young and Mandelstam 2013). Many of the headings are also justified, in their authority records, with reference to other reference sources from outside of the library world, such as film dictionaries and encyclopedias, and databases such as IMDb (www.imdb.com). Another key internationally-focused vocabulary is the Periodical Indexing Project (P.I.P) from the International Federation of Film Archives (FIAF) (https://www.fiafnet.org/images/tinyUpload/2020/04/Film_General_Subjects.docx). The wide range of sources used, published in different parts of the world and by quite different organisations, itself suggests an assumption in the universality of genre across time, space and domain.

Film studies is another field that, as well as library and information science, closely deals with conceptions and implementations of film genres. Bondebjerg (2015, p.160) states that ' $[\mathrm{g}]$ enre is a concept used in film studies and film theory to describe similarities between groups of films based on aesthetic or broader social, institutional, cultural, and psychological aspects.' The field of film studies has also tended to assume universal genres that everyone agrees on, with many film scholars making use of fixed film genre classifications in their research, regardless of the specific cultural, linguistic and temporal backdrop. For instance, research on which genres are more common in different parts of the world or across time has tended to assume that films are viewed and labelled the same way by different populations (Dauenhaurer et al., 2014; Follows, 
2018). However, this assumption has increasingly been called into question, with film scholars pointing out the culturally specific and fluid nature of genres (Tarancón, 2010; Tudor, 2012).

Although much of today's film industry is 'global', this does not mean that filmmakers, nor film audiences, use a set vocabulary. For commercial reasons, there may still be a strong Hollywood influence on genre, but there are many other influences, including other commercial ones (such as Bollywood) and including cultural ones. Even within cultures, the interplay of the different views and objectives of filmmakers, audiences, critics and educators adds layers of complexity to the way genres evolve as concepts and labels (Tudor, 2012). This interplay has also been studied in other fields, outside of film studies, and in relation to a range of genre types outside of film. In sociology, DiMaggio's framework (1987) for the study of art classification has been used to investigate its various social dimensions, including differentiation, hierarchy, universality and boundary strength. The framework also recognises the different impacts of bureaucracy, commerce and the mediating professions on genres or 'ritual classifications'. Meanwhile, genre theory has studied both the ways in which groups view genres and the ways in which groups are embodied in genres (Duff, 2000). All these studies have pointed to genres, including film genres, as dependent on both time and culture, but none has examined the degree to which differences are present within the same kind of professional classification, developed in different institutions in different parts of the English-speaking world. While the libraries of this world tend to follow the lead of the Library of Congress and use a shared taxonomy, i.e. the LCGFT, film institutes and archives have tended to develop their own classifications, now used to help organise their online collections and catalogues. The degree of alignment of these particular classifications is the subject of this study.

\section{Methodology}

Paradigmatically this work is derived from examination of third order classification theory (Tennis 2018). Third order classification theory, as discussed by Tennis (2018), looks at how knowledge organization systems relate to given populations and contexts. Studies in this area 'draw out both the differences and similarities between the universe of classification schemes (and their allied constructions in the realm of indexing languages), in order to enter into a reexamination of the nature and utility of particular schemes in particular contexts' (Tennis 2018, p. 705). This work is also an extension of research by Hider, White and Barlow (forthcoming) on film genre and the moving picture terms included in the Library of Congress Genre Form Terms.

While paradigmatically the same, this work is procedurally unlike Hider, White and Barlow (forthcoming) because it specifically compares three film genre vocabularies of similar functionality used in three different cultural contexts. One vocabulary from each culture was chosen for comparison. Data consisting of all the terms from the American Film Institute (AFI), Australia Centre for the Moving Image (ACMI), and British Film Institute (BFI) genre lists were extracted from their respective websites in early August 2019. Data analysis used descriptive statistical frequencies and quantitative mapping techniques to assess inter-alignment of the three vocabularies in six different coding instances. The six genre list comparisons were: BFI to ACMI; ACMI to BFI; BFI to AFI; AFI to BFI; AFI to ACMI; and ACMI to AFI. Coding development and multiple pre-testing are discussed in detail as follows. 
In late August to early September 2019, three rounds of pretesting were conducted between two coders in order to refine the code book and coding process, which aimed to gauge the degree of alignment between the vocabularies. The first test was conducted between 20 to 23 August 2019 using only one coding scale based on White's (2013) comparison of keywords and LCSH that included six codes. Intercoding consistency from pretest one resulted in $60.7 \%$ (17 out of 28 terms) agreement between the two coders. These results suggested that changes needed to be made to the coding scale, and it became clear that the use of two coding scales would work better, as they would avoid conflation of semantic and syntactic elements. The second pretest was conducted on 26 August 2019 using the two new scales. Agreement for the second pretest was much better at $94.6 \%$ (53 out of 56 terms). Modifications were made to the definitions of some of the codes, and a third test was conducted on 1 September 2019, resulting in $87.5 \%$ (49 out of 56 terms) agreement. The third test resulted in a decision to prioritize the semantic over the syntactic when a choice of mapped-to terms occurred (i.e. the term closer in meaning than in form was preferred). The final coding scales used for the study are described further below.

\section{Coding Scales}

Two coding scales were used to evaluate the overlap between the vocabularies. The first scale focused on meaning to evaluate the degree of semantic alignment. Figure 1 outlines the Meaning Scale coding and explains how each code should be applied. Codes range from 1 to 4, with 1 representing the closest alignment. The hierarchy of the schemes themselves were not considered on this scale, only the relationship between the intended meanings of the two terms, as interpreted by the coder. However, this interpretation in some instances involved reference to the scheme in which the terms were located (e.g. to broader or narrower terms).

Figure 1. Meaning Scale

\section{1: Equivalent relationship match}

Terms have the equivalent meaning.

\section{2: Hierarchical relationship match}

Terms are related in a hierarchical way, one broader than the other. (Ex. Political films vs Political satire films.)

\section{3: Associative relationship match}

Terms are related closely in a "sibling type" relationship. (Ex. Children in Film vs Children's movies.)

\section{4: No match}

Terms are not semantically related, in a way that one would expect to be indicated in a subject thesaurus, for example 
The second scale focused on word form to evaluate the degree of syntactic alignment. Codes range from 1 to 5, with 1 representing the closest alignment. Count was ignored throughout during matching, as were terms representing the concept of film. Figure 2 outlines the Word Form Scale.

Figure 2. Word Form Scale

\section{1: Perfect Match}

All words in the terms match exactly, including spelling, except for count, (e.g. sport and sports films; science fiction and science fiction movies).

\section{2: Orthographic Variant Match}

All words in the terms match exactly, except for count and spelling, (e.g. humor and humour).

\section{3: Word Form Variant Match}

All words in the terms share the same word stems, but differ in word form, beyond count and spelling (e.g. animation vs animated, science fiction vs sci-fi, romance comedies vs romantic comedies).

\section{4: Phrase Overlap Match}

At least one of the words in the terms match share the same word stem (and might match exactly; e.g. romantic comedies vs comedy).

\section{5: No Match}

None of the words in the terms share the same word stem.

After finalizing the coding scheme, the six rounds of research comparing the vocabularies were conducted in October 2019.

\section{Data Analysis}

Quantitative data analysis techniques based on basic statistical frequencies were used to examine three areas: 1 . to identify unique terms to each vocabulary; 2 . to identify unique matches between two vocabularies or shared unique terms; 3 . to examine the mean, median, and percentage match between each vocabulary according to both meaning and word form. Unique terms to a vocabulary is when terms (based mainly on semantic matches) occur within one vocabulary. Unique matches between two vocabularies, or shared unique terms, is when a term in one vocabulary has a semantic match in only one other vocabulary. The Results and Analysis section that follows discusses the findings in these three areas for three vocabularies from the Australian Centre for Moving Images, the British Film Institute, and the American Film Institute.

\section{Results and Analysis}


The results of this research expand on the research done by Hider, White and Barlow (forthcoming) by comparing three film genre vocabularies: those of the Australian Centre for Moving Images (ACMI), the British Film Institute (BFI) and American Film Institute (AFI). For the rest of this paper, the vocabularies from the ACMI, BFI and AFI may be referred to by their institutional abbreviation listing. General characteristics of each vocabulary will be discussed prior to presenting the analysis of unique terms, unique matches between vocabularies, and meaning and word form matches.

\section{Australian Centre for Moving Images (ACMI)}

The vocabulary used by the Australian Centre for Moving Images (ACMI) had 180 terms related to film genre, containing substantially more terms than those used by the BFI (28 terms) and AFI (29 terms). The ACMI is Australia's national museum of film, $\mathrm{TV}$, video games, and digital culture and art. The genre classification appears to be based on 'literary' or collection warrant, whereby the terms listed are based on items that exist within the collection. The terms are presented with hierarchical relationships between main and sub-genres. The format word 'films' is used as part of 100 out of the 181 terms (55.2\%). One term uses the format word 'movies' ('Road movies', a subterm of 'Drama'). The term 'cinema' is also used once (in 'Gay/Lesbian (Queer Cinema)').

ACMI vocabulary was the base vocabulary in two rounds of comparisons: ACMI to BFI and ACMI to AFI. The vocabulary had a total of 33 unique terms $(18.3 \%)$ that had no matches to the other two vocabularies. Considering that ACMI vocabulary was much larger than the other two vocabularies, it is perhaps surprising that a little less than $20 \%$ of terms were unique.

The ACMI vocabulary had 61 terms (33.9\%) that only mapped to BFI (and not AFI). A total of 43 out of those 61 matches (70.5\%) related to Animation, a concept not found in the AFI vocabulary. A total of 53 terms (29.4\%) did not map to BFI.

The ACMI vocabulary had 22 terms (12.2\%) that only mapped to AFI (and not BFI). A total of 8 out of those 22 matches $(36.4 \%)$ related to Experimental Films. A total of 93 terms $(51.7 \%)$ did not map to AFI.

Table 1 shows ACMI-based Vocabulary Matching scores for the two rounds comparing ACMI to BFI and ACMI to AFI in terms of Meaning Scale and Word Form Scale. Out of the ACMI terms, 126 terms (70.0\%) matched to BFI terms on the Meaning Scale with a score of 1,2 , or 3, whereas only 87 terms $(48.3 \%)$ matched to AFI terms with these scores.

Table 1. ACMI-based Vocabulary Matching

\begin{tabular}{|c|c|c|c|c|}
\hline \multirow[b]{2}{*}{ Meaning Scale (1-4) } & \multicolumn{2}{|c|}{$\begin{array}{l}\text { ACMI to BFI } \\
(180)\end{array}$} & \multicolumn{2}{|c|}{$\begin{array}{l}\text { ACMI to AFI } \\
(180) \quad(29)\end{array}$} \\
\hline & Frequency & Percentage & Frequency & Percentage \\
\hline 1 - Equivalent & 10 & $5.56 \%$ & 17 & $9.44 \%$ \\
\hline
\end{tabular}




\begin{tabular}{|c|c|c|c|c|}
\hline 2 - Hierarchical & 92 & $51.11 \%$ & 69 & $38.33 \%$ \\
\hline 3 - Associative & 24 & $13.33 \%$ & 1 & $0.56 \%$ \\
\hline 4 - No match & 54 & $30.00 \%$ & 93 & $51.67 \%$ \\
\hline Median & \multicolumn{2}{|c|}{2} & \multicolumn{2}{|c|}{4} \\
\hline Mean & \multicolumn{2}{|c|}{2.7} & \multicolumn{2}{|c|}{2.9} \\
\hline Frequency total 1-3 & \multicolumn{2}{|c|}{126} & \multicolumn{2}{|c|}{87} \\
\hline Percentage of total & \multicolumn{2}{|c|}{$70.00 \%$} & \multicolumn{2}{|c|}{$48.33 \%$} \\
\hline & \multicolumn{2}{|c|}{$\begin{array}{l}\text { ACMI to BFI } \\
(180)\end{array}$} & \multicolumn{2}{|c|}{$\begin{array}{l}\text { ACMI to AFI } \\
\begin{array}{cc}(180) & (29)\end{array}\end{array}$} \\
\hline Word Form Scale (1-5) & Frequency & Percentage & Frequency & Percentage \\
\hline 1 - Perfect match & 9 & $5.00 \%$ & 18 & $10.00 \%$ \\
\hline 2 - Orthographic & 1 & $0.56 \%$ & 0 & $0.0 \%$ \\
\hline $3-$ Word form & 1 & $0.56 \%$ & 2 & $1.11 \%$ \\
\hline 4 - Phrase overlap & 94 & $52.22 \%$ & 57 & $31.67 \%$ \\
\hline 5 - No match & 75 & $41.67 \%$ & 103 & $57.22 \%$ \\
\hline Median & \multicolumn{2}{|c|}{4} & \multicolumn{2}{|c|}{5} \\
\hline Mean & \multicolumn{2}{|c|}{4.5} & \multicolumn{2}{|c|}{4.3} \\
\hline Frequency total 1-4 & \multicolumn{2}{|c|}{105} & \multicolumn{2}{|c|}{77} \\
\hline Percentage of total & \multicolumn{2}{|c|}{$58.33 \%$} & \multicolumn{2}{|c|}{$42.78 \%$} \\
\hline
\end{tabular}

\section{British Film Institute (BFI)}

The vocabulary used by the British Film Institute (BFI) has a total of 28 terms presented as a list with no interrelations indicated between terms. It is used by the BFI to assign primary genres to every film released in the United Kingdom. The format terms representing the concept of film are inconsistently used in the vocabulary. The words 'pictures', 'cinema', 'film' and 'films' are all used throughout without any apparent distinction. 
BFI vocabulary was the base vocabulary in two rounds of comparisons: BFI to ACMI and BFI to AFI. The BFI vocabulary had a total of 9 out of 28 unique terms $(32.1 \%)$ that had no matches to either of the other vocabularies. Four of those terms have 'British' as one of the words that make up the term: 'Asian-British Cinema', 'Black British Film', 'British Pioneers', and 'British-Chinese Cinema'. The remaining five unique terms were 'B Pictures', 'Short Films', 'Social Problem Films', 'Social Realism' and 'Women and Film'.

The BFI vocabulary had 8 terms (28.6\%) that only mapped to ACMI (and not AFI). Those terms were 'Costume Drama', 'Early Spy Films', 'Gangsters', 'Literary Adaptation', 'Newsreels', 'Political Films', 'Public Information Fillers' and 'Thriller'. A total of 9 BFI terms $(32.1 \%)$ did not map to ACMI.

BFI had no terms that mapped only to AFI (and not ACMI). A total of 18 BFI terms $(64.3 \%)$ had no matches whatsoever in the AFI vocabulary. This suggests a relative distance between these two vocabularies.

Table 2 shows BFI-based Vocabulary Matching scores for the two rounds comparing BFI to ACMI and BFI to AFI, in terms of both Meaning Scale and Word Form Scale. When compared to ACMI, 19 terms (67.9\%) matched on the Meaning Scale with a score of 1,2 , or 3 ; in comparison to AFI, 8 terms (30.8\%) matched.

Table 2. BFI-based Vocabulary Matching

\begin{tabular}{|c|c|c|c|c|}
\hline & \multicolumn{2}{|c|}{$\begin{array}{l}\text { BFI to ACMI } \\
(28) \quad(180)\end{array}$} & \multicolumn{2}{|c|}{$\begin{array}{l}\text { BFI to AFI } \\
\begin{array}{lr}(28) & (29)\end{array}\end{array}$} \\
\hline Meaning Scale(1-4) & Frequency & Percentage & Frequency & Percentage \\
\hline 1 - Equivalent & 12 & $42.86 \%$ & 6 & $21.43 \%$ \\
\hline 2 - Hierarchical & 6 & $21.43 \%$ & 0 & $0.0 \%$ \\
\hline 3 - Associative & 1 & $3.57 \%$ & 2 & $7.14 \%$ \\
\hline 4 - No match & 9 & $32.10 \%$ & 18 & $64.29 \%$ \\
\hline Median & \multicolumn{2}{|c|}{2} & \multicolumn{2}{|c|}{4} \\
\hline Mean & \multicolumn{2}{|c|}{2.3} & \multicolumn{2}{|c|}{3.0} \\
\hline Frequency total 1-3 & \multicolumn{2}{|c|}{19} & \multicolumn{2}{|c|}{8} \\
\hline Percentage of total & \multicolumn{2}{|c|}{$67.86 \%$} & \multicolumn{2}{|c|}{$28.57 \%$} \\
\hline & \multicolumn{2}{|c|}{$\begin{array}{l}\text { BFI to ACMI } \\
(28) \quad(180)\end{array}$} & \multicolumn{2}{|c|}{$\begin{array}{l}\text { BFI to AFI } \\
\begin{array}{ll}(28) & (29)\end{array}\end{array}$} \\
\hline
\end{tabular}




\begin{tabular}{|c|c|c|c|c|}
\hline Word Form Scale (1-5) & Frequency & Percentage & Frequency & Percentage \\
\hline 1 - Perfect match & 10 & $35.71 \%$ & 6 & $21.43 \%$ \\
\hline 2 - Orthographic & 0 & $0.0 \%$ & 0 & $0.0 \%$ \\
\hline $3-$ Word form & 1 & $3.57 \%$ & 0 & $0.0 \%$ \\
\hline 4 - Phrase overlap & 5 & $17.86 \%$ & 2 & $7.14 \%$ \\
\hline 5 - No match & 12 & $42.86 \%$ & 20 & $71.43 \%$ \\
\hline Median & \multicolumn{2}{|c|}{4} & \multicolumn{2}{|c|}{5} \\
\hline Mean & \multicolumn{2}{|c|}{3.32} & \multicolumn{2}{|c|}{4.07} \\
\hline Frequency total 1-4 & \multicolumn{2}{|c|}{16} & \multicolumn{2}{|c|}{8} \\
\hline Percentage of total & \multicolumn{2}{|c|}{$57.14 \%$} & \multicolumn{2}{|c|}{$28.57 \%$} \\
\hline
\end{tabular}

\section{American Film Institute (AFI)}

Similar to the BFI vocabulary, the American Film Institute (AFI) vocabulary has a total of 29 terms presented in a flat list with no interrelations indicated between terms. The AFI genre vocabulary is used in the AFI film database. Unlike the BFI vocabulary, the concept of 'film' is assumed in all the terms, with all the words within each term relating only to genre and not format.

AFI vocabulary was the base vocabulary in two rounds of comparisons: AFI to BFI and AFI to ACMI. The AFI vocabulary had 4 out of 29 terms (13.8\%) with no matches at any level to the other vocabularies. Those four terms had nothing obviously in common and were 'Allegory', 'Anthology', 'Epic' and 'Variety'.

The AFI vocabulary had 7 terms (24.1\%) that only mapped to ACMI (and not BFI). Those terms were 'Adventure', 'Biography', 'Fantasy', 'Film Noir', 'Mystery', 'Swashbuckler' and 'Western'. A total of 4 terms (13.8\%) did not map to ACMI.

Similar to findings from the BFI-based rounds, the AFI vocabulary had no unique matches with the BFI vocabulary. A total of 11 terms $(38.0 \%)$ did not map to BFI. Again, this suggests a relative distance between AFI and BFI vocabularies.

Table 3 shows AFI-based Vocabulary Matching scores for the two rounds comparing AFI to BFI and AFI to ACMI in terms of Meaning Scale and Word Form Scale. When compared to BFI, 18 terms $(62.1 \%)$ matched on the Meaning Scale with a score of 1, 2, or 3; in comparison to ACMI, 25 terms (86.2\%) matched.

Table 3. AFI-based Vocabulary Matching 


\begin{tabular}{|c|c|c|c|c|}
\hline \multirow[b]{2}{*}{ Meaning Scale (1-4) } & \multicolumn{2}{|c|}{$\begin{array}{l}\text { AFI to BFI } \\
(29) \quad(28)\end{array}$} & \multicolumn{2}{|c|}{$\begin{array}{l}\text { AFI to ACMI } \\
\begin{array}{ll}(29) & (180)\end{array}\end{array}$} \\
\hline & Frequency & Percentage & Frequency & Percentage \\
\hline 1 - Equivalent & 9 & $31.03 \%$ & 20 & $68.97 \%$ \\
\hline 2 - Hierarchical & 8 & $27.59 \%$ & 4 & $13.79 \%$ \\
\hline 3 - Associative & 1 & $3.45 \%$ & 1 & $3.45 \%$ \\
\hline 4 - No match & 11 & $37.93 \%$ & 4 & $13.79 \%$ \\
\hline Median & \multicolumn{2}{|c|}{2} & \multicolumn{2}{|c|}{1} \\
\hline Mean & \multicolumn{2}{|c|}{2.5} & \multicolumn{2}{|c|}{1.6} \\
\hline Frequency total 1-3 & \multicolumn{2}{|c|}{18} & \multicolumn{2}{|c|}{25} \\
\hline Percentage of total & \multicolumn{2}{|c|}{$62.07 \%$} & \multicolumn{2}{|c|}{$86.21 \%$} \\
\hline & \multicolumn{2}{|c|}{$\begin{array}{l}\text { AFI to BFI } \\
(29) \quad(28)\end{array}$} & \multicolumn{2}{|c|}{$\begin{array}{l}\text { AFI to BFI } \\
(29) \quad(28)\end{array}$} \\
\hline Word Form Scale (1-5) & Frequency & Percentage & Frequency & Percentage \\
\hline 1 - Perfect match & 6 & $20.69 \%$ & 18 & $62.07 \%$ \\
\hline 2 - Orthographic & 0 & $0.0 \%$ & 0 & $0.0 \%$ \\
\hline $3-$ Word form & 0 & $0.0 \%$ & 1 & $3.45 \%$ \\
\hline 4 - Phrase overlap & 9 & $31.03 \%$ & 6 & $20.69 \%$ \\
\hline 5 - No match & 14 & $48.28 \%$ & 4 & $13.79 \%$ \\
\hline Median & \multicolumn{2}{|c|}{4} & \multicolumn{2}{|c|}{1} \\
\hline Mean & \multicolumn{2}{|c|}{3.86} & \multicolumn{2}{|c|}{2.24} \\
\hline Frequency total 1-4 & \multicolumn{2}{|c|}{15} & \multicolumn{2}{|c|}{25} \\
\hline Percentage of total & \multicolumn{2}{|c|}{$51.72 \%$} & \multicolumn{2}{|c|}{$86.21 \%$} \\
\hline
\end{tabular}

\section{Comparing across all three vocabularies}


The three vocabularies shared five terms that were directly equivalent, both semantically and syntactically: 'Comedy', 'Documentary', 'Horror', 'Melodrama', and 'Musicals' had 'perfect' mapping scores. There also five other terms that were semantically equivalent across the three vocabularies: children's films, performance, science fiction, experimental film, and war. These 10 common terms contrast with 46 terms that were unique to one or other of the vocabularies. In other words, there were more genres that were not shared than shared.

Looking at the six one-to-one comparisons together, a picture emerges of the three vocabularies' relative semantic and syntactic proximity. Table 4 shows the average scores for the six mappings, in relation to the vocabulary Meaning Scale.

Table 4. Most to Least Matching in Relation to Meaning

\begin{tabular}{|l|l|c|c|}
\hline & Vocabulary Match & Median & Mean \\
\hline Most Related & AFI to ACMI & 1 & 1.6 \\
\hline & BFI to ACMI & 2 & 2.3 \\
\cline { 2 - 4 } & AFI to BFI & 2 & 2.5 \\
\cline { 2 - 4 } & ACMI to BFI & 2 & 2.7 \\
\cline { 2 - 4 } & ACMI to AFI & 4 & 2.9 \\
\hline Least Related & BFI to AFI & 4 & 3.0 \\
\hline
\end{tabular}

The AFI to ACMI comparison was the closest mapping, with a median score of 1 and a mean score of 1.62. The least close mapping was BFI to AFI, with a median score of 4 and a mean score of 3.0. When used as the base vocabulary, both the AFI and BFI vocabularies mapped well to the ACMI vocabulary. The poorer mapping to both the AFI and BFI vocabularies when the ACMI vocabulary is the base vocabulary may be due in part to the greater size of the ACMI vocabulary. Overall, the scores from the mappings between each of the three pairs of vocabularies suggest that ACMI is quite close to both AFI and BFI, semantically, and that AFI and BFI are less close. This ties in with the results reported in the vocabulary specific sections.

Table 5 shows the average scores for the six mappings in relation to the vocabulary Word Form Scale. In general, word form scores were lower than the meaning scores, but the order of the mappings was similar, with the AFI to ACMI comparison again the closest mapping, with a median score of 1 and a mean score of 2.24, even though the BFI and ACMI vocabularies share British English spellings. In fact, across all vocabulary matching rounds the Word Form code 2-Orthographic Variant Match was, somewhat unexpectedly, not used. 
Table 5. Most to Least Matching in Relation to Word Form

\begin{tabular}{|l|l|c|c|}
\hline & Vocabulary Match & Median & Mean \\
\hline Most Related & AFI to ACMI & 1 & 2.24 \\
\hline & BFI to ACMI & 4 & 3.32 \\
\cline { 2 - 4 } & AFI to BFI & 4 & 3.86 \\
\cline { 2 - 4 } & BFI to AFI & 5 & 4.07 \\
\cline { 2 - 4 } & ACMI to BFI & 4 & 4.5 \\
\hline Least Related & ACMI to AFI & 5 & 4.26 \\
\hline
\end{tabular}

\section{Conclusion}

The findings of this study point to a fair degree of overlap between the three film vocabularies, as one would expect to observe among classifications developed by institutions with similar functions, albeit in different countries and with somewhat different collections. Clearly film genre is international, especially in the Englishspeaking world, with commercial and artistic interests that stretch well beyond national boundaries, and increasingly so in the digital world. On the other hand, the findings also point to a significant degree of difference across the three film institutes, which is likely due to a combination of factors: cultural-specific genre traditions and emphases, local institutional and curatorial conditions and histories, and collection differences.

The English-speaking library cataloguing world for the most part adheres to international standards that assume the universality of concepts such as subjects and genres. These standards include the American-based Library of Congress vocabularies of LCSH and LCGFT. Yet just as LCSH have been criticised, including by Australian librarians, for their US-centricity, Hider, White and Barlow (forthcoming) found that LCGFT were more closely aligned to the AFI vocabulary than either ACMI or BFI. On the other hand, Hider, White and Barlow (forthcoming) also note that the only terms that appear in the six vocabularies they studied, including LCGFT and the those of the three film institutions, are 'Horror' and 'Comedy', while overall their study points to a significant degree of non-alignment between the library-oriented LCGFT and all three vocabularies supporting specialized film curation. Although library cataloguing and film curation serve slightly different ends, these two studies raise the question as to how universalist the approach of Anglo-American cataloguing truly is, and whether it can learn more from the various approaches to film genre, for instance, that can be found around the curatorial world.

In terms of alignment across the three film institutions' vocabularies, that of ACMI appears to sit roughly in the middle, between the American and British vocabularies. The ACMI vocabulary has term matches with both the AFI and BFI vocabularies, whereas the AFI and BFI do not share any term matches. This may be partly due to the much larger size of the ACMI vocabulary, but the average scores 
suggest that that Australian film language intersects the American and British film languages, as an historical perspective of Australian cinema might predict. However, more research into the interrelations between Australian, American and British film cultures is needed in order to explore this intersection in more depth.

\section{Bibliography}

Bondebjerg, I. (2015). “Film: Genre and genre theory.” In Wright, J. D. (Ed)., International encyclopedia of the social \& behavioral sciences. 2 ed. Amsterdam: Elsevier.

Dauenhauer, J., Hockett, J., Mammarelli, J. \& Yarem, M. (2014). Information analysis of movie genres. Philadelphia: Drexel University.

http://cluster.ischool.drexel.edu/ cchen/courses/INFO633/13-14/mammarelli.pdf

DiMaggio, P. (1987). Classification in art. American Sociological Review 52(8), 440-55.

Duff, D., (Ed.),(2000). Modern genre theory. Harlow, Essex: Longman.

Follows, S. (2018, February 19). How the genre of film production changes around the world. Stephen Follows Film and Data Education. Available at: https://stephenfollows.com/genrefilm-production-changes-around-world/

Hider, P. (2019). From Anderson to ORDAC: A history of bibliographic policy discussion in Australia. Journal of the Australian Library and Information Association, 68(1), 3-17.

Hider, P., White, H., \& Barlow, P. (accepted/forthcoming Feb 2021). Film Genre and Everyday Vocabularies: Relationships between Everyday Vocabularies and the Library of Congress Genre Form Terms. Library Trends, 69(3).

Library of Congress. (2019). Introduction. In Library of Congress Genre/Form Terms for Library and Archival Materials, 1-7.

McKinlay, J. (1979). List of Australian subject headings: Too little, too late? Australian Library Journal, 28(2), 23-26.

Tarancón, J. A. (2010). Genre matters: Film criticism and the social relevance of genres, Cineaction

Tennis, J. T. (2018). Four orders of classification theory and their implications, Cataloging \& Classification Quarterly 56(6), 702-10.

Tudor, A. (2012). Genre. In Grant, B.K.(Ed.), Film genre reader IV. University of Texas Press.

White, H. (2013). "Examining Scientific Vocabulary: Mapping Controlled Vocabularies with Free Text Keywords." Cataloging \& Classification Quarterly 51(6), 655-74.

Young, J L., \& Mandelstam, Y. (2013). "It takes a Village: Developing Library of Congress Genre/Form Terms." Cataloging \& Classification Quarterly 51(1-3), 6-24. 